\title{
Orientación empática de estudiantes de medicina en tres universidades de Barranquilla, Colombia y en una universidad de República Dominicana
}

\author{
Empathic orientation among medical students from three \\ universities in Barranquilla, Colombia and one university in \\ the Dominican Republic
}

\begin{abstract}
Dr. Víctor Patricio Díaz Narváez ${ }^{a}$, Dra. Luz Marina Alonso Palaciob,
Mg. Sara Elvira Caro ${ }^{b}$, Dra. María Guadalupe Silvac , Mg. Joel Arboleda Castilloc,

Dr. Jorge Luis Bilbao y Dr. Jesús Iglesias Acosta
\end{abstract}

a. Departamento de Postgrado. Facultad de Odontología. Universidad Finis Terrae. Santiago de Chile.

b. División Ciencias de la Salud. Universidad del Norte. Barranquilla. Colombia.

c. Instituto de Investigaciones Científicas. Universidad Central del Este. República Dominicana.

d. Facultad de Medicina.

Universidad Libre Seccional Barranquilla y Fundación Universitaria San Martín Sede Puerto Colombia. Barranquilla. Colombia.

e. Facultad Ciencias de la Salud.

Universidad Libre Seccional Barranquilla. Colombia.

Correspondencia: Dr. Víctor Patricio Díaz Narváez: victor.diaz@ umayor.cl

Conflicto de intereses: Ninguno que declarar.

Recibido: 26-4-2013 Aceptado: 29-7-2013

\section{RESUMEN}

Objetivo. Comparar la orientación empática entre estudiantes de Medicina de tres facultades de Medicina de Colombia y una de República Dominicana.

Material y métodos. Se midió la orientación empática de los estudiantes de Medicina mediante la escala de empatía médica de Jefferson, en la versión en español para estudiantes (versión S) validada en México y Chile, y adaptada culturalmente a Colombia y República Dominicana. Los datos se compararon mediante un análisis de varianza trifactorial (modelo III) y por un análisis discriminante. Resultados. No se encontraron diferencias de orientación empática entre los cursos ni entre los sexos, pero sí entre las facultades de Medicina consideradas como una unidad en cada uno de los países estudiados.

Conclusiones. Los niveles de orientación empática tienden a disminuir a medida que los cursos avanzan. Este hecho se produce en estudiantes de ambos sexos y en todas las facultades analizadas.

Palabras clave:empatía, escala de empatía médicade Jefferson (EEMJ), orientación empática, componentes de la empatía.

http://dx.doi.org/10.5546/aap.2014.e41

\section{INTRODUCCIÓN}

Hojat y cols., ${ }^{1}$ plantean que la empatía es un concepto de tal amplitud, que lo hace pasible de diferentes definiciones, enfoques y teorías. ${ }^{1,2}$ Así, puede explicársela como: a) cogniti$\mathrm{va}^{3,4} \mathrm{o}$ b) cognitiva y emocional, de incluirse la inteligencia emocional para su análisis. ${ }^{5,6}$ La habilidad para comprender la experiencia y los senti- mientos de otras personas, y la capacidad de observar y entender el mundo desde la perspectiva del otro están relacionadas con el componente cognitivo; ${ }^{7}$ a su vez, el aspecto afectivo está relacionado con la aptitud de unirse a las experiencias de otros. ${ }^{7,8}$ Existen estudios que demuestran que la presencia de empatía en los médicos permite desarrollar ventajas para una mejor atención del paciente desde que:

a) aumenta su satisfacción, ${ }^{9}$

b) incrementa su complacencia, ${ }^{10}$

c) acrecienta la habilidad del médico para el diagnóstico y tratamiento, ${ }^{11} \mathrm{y}$

d) baja significativamente el riesgo de juicio por mala práctica ${ }^{12}$ y es transversal a todas las especialidades.

Cuando la relación médicopaciente adquiere carácter positivo, la posibilidad de curación crece sustancialmente y constituye, por lo tanto, uno de los elementos críticos en la práctica médica. ${ }^{13,14}$ La creciente mediación de la tecnología produce consecuencias: los pacientes perciben a sus médicos distantes de ellos. ${ }^{15}$ El componente empático resulta importante en la formación de los estudiantes de Medicina y, por ende, debería ser incorporado al proceso de enseñanza-aprendizaje. La empatía se ha estudiado en Europa, Estados 
Unidos e Inglaterra (entre otros países), ${ }^{1-5}$ pero hasta donde conocemos, no existe literatura reportada sobre cómo opera la empatía en los estudiantes de Medicina en el norte de América del Sur y en El Caribe, ni trabajos que comparen facultades de un mismo país o países diferentes. Nuestro objetivo fue evaluar y comparar la orientación empática de los estudiantes de Medicina de cuatro universidades: tres de la ciudad de Barranquilla (Colombia) y una de República Dominicana.

\section{POBLACIÓN Y MÉTODO}

Este trabajo, de tipo exploratorio, no experimental, descriptivo, de corte transversal y ex post facto causa-efecto, regido bioéticamente por las normas de Helsinski, aborda poblaciones compuestas por los estudiantes pertenecientes a los niveles primero, segundo, tercero, cuarto y quinto año de la Carrera de Medicina de las Facultades de Medicina de la Universidad del Norte, Universidad San Martín y Universidad Libre, situadas en la ciudad de Barranquilla (Colombia) y la Universidad Central del Este de la República Dominicana. La recolección de datos se realizó en tres semanas, desde el 26 de marzo hasta el 13 de abril de 2012, de forma casi simultánea en todas las facultades señaladas (de todos los países involucrados). La muestra la constituyeron todos aquellos sujetos que pudieron ser evaluados en el día que les correspondía. Todos los estudiantes, en el momento de la aplicación de la escala, estaban cursando el último tramo del primer semestre de cada curso. A los participantes de cada una de las muestras se les aplicó la escala de empatía médica de Jefferson (EEMJ) en la versión en español para estudiantes (versión S), validada en México y Chile, ${ }^{13,15}$ y adaptada culturalmente a Colombia y República Dominicana mediante Criterio de Jueces (comisión de jueces nombrados en Barranquilla, Colombia, y en República Dominicana para tal efecto) (Anexo 1). No existieron criterios de exclusión, pues el objeto era evaluar la variable de interés a la mayor cantidad de estudiantes. En congruencia con lo antes referido, se realizó una única medición anónima y confidencial, mediante un operador neutral, a los alumnos de primero a cuarto año en salas de clases. En el caso de los estudiantes de quinto año, se administró el instrumento en una visita a las instalaciones clínicas, con las mismas indicaciones señaladas previamente.

\section{Análisis estadístico}

Las sumatorias del puntaje de los datos primarios obtenidos en la escala antes referida fueron sometidas inicialmente a la prueba de normalidad de Shapiro-Wilk y homocedasticidad de Leveneen los tres factores estudiados (universidad, año y género). ${ }^{16,17}$ Se estimaron los estadígrafos descriptivos; media aritmética y desviación típica en todos los factores y sus niveles correspondientes. La comparación de las medias dentro de los niveles de los factores principales estudiados (universidad, curso y género) se realizaron mediante un modelo lineal general univariado (ANOVA) trifactorial, modelo III, y una prueba de comparación múltiple de Duncan, para datos desbalanceados en concordancia con Díaz. ${ }^{18}$ Se evaluó la potencia observada (1- $\beta$ ) y el tamaño del efecto. ${ }^{18,19}$ Los estadígrafos estimados se dibujaron en un gráfico aritmético simple. Posteriormente se realizó un análisis discriminante, sobre la base de los valores de las respuestas de cada pregunta contenida en la escala EEMJ, tomadas en conjunto como variables independientes. Se empleó el estadígrafo $\lambda$ de Wilks. ${ }^{20}$ Para comprobar si las matrices de varianzas y covarianzas de cada universidad (Facultad de Medicina) provenían o no de una misma población, se empleó la prueba $\mathrm{M}$ de Box. ${ }^{21}$ Se estimaron los autovalores, ${ }^{17}$ así como la correlación canónica. ${ }^{20}$ Los centroides de los grupos se presentaron en gráficos. Por último, se ponderó el porcentaje de datos correctamente clasificados por universidad y en la totalidad del análisis realizado. Los datos se procesaron mediante el programa estadístico SPSS 20.0. El nivel de significación utilizado fue de $\alpha \leq 0,05 \mathrm{y}$ $\beta \leq 0,05$ en todos los casos.

\section{RESULTADOS}

Participaron alumnos de las Facultades de Medicina de la Universidad del Norte $(n=345 / 859$; $40,16 \%)$, Universidad San Martín ( $n=283 / 493$; $57,4 \%)$ y Universidad Libre $(n=695 / 1129$; $61,56 \%$ ), de la ciudad de Barranquilla (Colombia), y la Universidad Central del Este $(n=515 / 853$; 60,38\%) de República Dominicana. Ningún estudiante se opuso a la realización de la encuesta. En la Tabla 1 se presentan los resultados de la aplicación del modelo trifactorial. Se observó que los factores "universidad", "género" y la interacción entre los factores "universidad" (Facultad de Medicina) y "curso" (año) fueron altamente significativos $(p=0,005)$. Sin embargo, el estadígrafo $\eta^{2}$, que indica el tamaño del efecto del "tratamiento" 
estudiado, es pequeño. La potencia de la prueba, en todos los casos, está por encima del mínimo admisible: 0,95 (0,99 en general). Todo lo anterior muestra que los efectos de los factores nombrados y de su interacción son pequeños, pero no existe riesgo de cometer el error de tipo II. Como consecuencia, las diferencias o similitudes encon- tradas deben ser examinadas con cautela, en el sentido de que existen, pero son de pequeña magnitud $\left(R^{2}=0,069\right)$. En las Tablas 2 y 3 se presentan los valores de las medias de las universidades y cursos estudiados, y la prueba de Duncan. Se observa que los niveles de empatía son mayores en la Universidad Libre y en la del Norte y meno-

TABLA 1. Resultados de la aplicación del modelo escogido para comparar las medias de los factores estudiados

\begin{tabular}{|c|c|c|c|c|c|c|c|}
\hline Origen & $\begin{array}{c}\text { Suma de cuadrados } \\
\text { tipo III }\end{array}$ & gl & $\begin{array}{c}\text { Media } \\
\text { cuadrática }\end{array}$ & $\mathbf{F}$ & Significancia & $\begin{array}{c}\text { Eta al cuadrado } \\
\text { parcial }\end{array}$ & $\begin{array}{c}\text { Potencia } \\
\text { observada }^{\mathrm{b}}\end{array}$ \\
\hline Modelo corregido & $34514,078^{a}$ & 39 & 884,976 & 4,468 & 0,000 & 0,088 & 1,000 \\
\hline Universidad & 4664,174 & 3 & 1554,725 & 7,850 & 0,000 & 0,013 & 0,990 \\
\hline Curso & 1681,534 & 4 & 420,383 & 2,122 & 0,076 & 0,005 & 0,633 \\
\hline Género & 4580,059 & 1 & 4580,059 & 23,125 & 0,000 & 0,013 & 0,998 \\
\hline Universidad* Curso & 8660,875 & 12 & 721,740 & 3,644 & 0,000 & 0,024 & 0,999 \\
\hline Universidad* Género & 352,875 & 3 & 117,625 & 0,594 & 0,619 & 0,001 & 0,175 \\
\hline Curso* Género & 1792,010 & 4 & 448,003 & 2,262 & 0,060 & 0,005 & 0,665 \\
\hline Universidad* Curso* & 2356,093 & 12 & 196,341 & 0,991 & 0,455 & 0,007 & 0,593 \\
\hline \multicolumn{8}{|l|}{ Género } \\
\hline Error & 356113,145 & 1798 & 198,061 & & & & \\
\hline Total & $2,080 \mathrm{E} 7$ & 1838 & & & & & \\
\hline Total corregida & 390627,223 & 1837 & & & & & \\
\hline
\end{tabular}

a. R. cuadrado=0,088 ( $\mathrm{R}$ cuadrado corregida $=0,069)$.

b. Calculado con alfa $=0,05$.

gl: grados de libertad.

TABLA 2. Resultados de la estimación de la media y el error típico de las sumatorias de los valores observados en cada universidad y en cada año analizado

\begin{tabular}{|c|c|c|c|c|c|}
\hline \multirow[t]{2}{*}{ Universidad } & \multirow[t]{2}{*}{ Cursos } & \multirow[t]{2}{*}{ Media } & \multirow[t]{2}{*}{ Error típico } & \multicolumn{2}{|c|}{ Intervalo de confianza $95 \%$} \\
\hline & & & & Límite inferior & Límite superior \\
\hline \multirow{5}{*}{$\begin{array}{l}\text { Universidad } \\
\text { del Norte }\end{array}$} & Primer año & 105,830 & 1,317 & 103,247 & 108,413 \\
\hline & Segundo año & 107,830 & 1,643 & 104,312 & 110,755 \\
\hline & Tercer año & 105,767 & 4,233 & 97,464 & 114,069 \\
\hline & Cuarto año & 110,711 & 2,199 & 106,399 & 115,023 \\
\hline & Quinto año & 106,100 & 2,360 & 101,471 & 110,729 \\
\hline \multirow{5}{*}{$\begin{array}{l}\text { Universidad } \\
\text { Libre }\end{array}$} & Primer año & 109,351 & 0,853 & 107,678 & 111,024 \\
\hline & Segundo año & 108,800 & 0,980 & 106,879 & 110,722 \\
\hline & Tercer año & 101,211 & 2,314 & 96,671 & 105,750 \\
\hline & Cuarto año & 102,129 & 1,343 & 99,495 & 104,763 \\
\hline & Quinto año & 101,964 & 2,452 & 97,155 & 106,773 \\
\hline Universidad & Primer año & 98,200 & 3,634 & 91,073 & 105,327 \\
\hline \multirow[t]{4}{*}{ San Martín } & Segundo año & 106,301 & 1,545 & 103,271 & 109,331 \\
\hline & Tercer año & 99,859 & 2,005 & 95,926 & 103,792 \\
\hline & Cuarto año & 104,553 & 1,661 & 101,295 & 107,811 \\
\hline & Quinto año & 102,340 & 1,906 & 98,603 & 106,077 \\
\hline Universidad & Primer año & 100,937 & 1,436 & 98,120 & 103,755 \\
\hline \multirow[t]{4}{*}{ Central del Este } & Segundo año & 99,490 & 1,154 & 97,226 & 101,754 \\
\hline & Tercer año & 101,843 & 1,444 & 99,012 & 104,675 \\
\hline & Cuarto año & 103,213 & 1,473 & 100,324 & 106,102 \\
\hline & Quinto año & 101,604 & 1,962 & 97,757 & 105,451 \\
\hline
\end{tabular}


res en la Universidad de San Martín, seguida de la Universidad Central del Este. En la Tabla 4 se presentan los resultados de la prueba de Duncan en los cursos. Se observó una disminución de los valores de la empatía a medida que aumentaba el curso estudiado y esto ocurrió en ambos sexos en todas las facultades estudiadas (Figuras 1 y 2). Los resultados observados en los $\lambda$ de Wilks, prueba M de Box, autovalor y regresión canónica, y $\chi^{2}$ permiten aplicar con confianza la prueba discriminante para comparar las matrices completas de las respuestas de la escala. En la Figura 3 se muestran los centroides de cada universidad estudiada. En dicha figura queda establecido que la Universidad Central del Este de República Dominicana se separa y distingue de las universidades de la ciudad de Barranquilla, Colombia. Por último, en la Tabla 5 se muestran los resultados de la clasificación que realizó el análisis discriminante de los sujetos en los grupos (universidades) examinados. Los estudiantes de la Universidad del Norte fueron clasificados correctamente en un $20 \%$. En la Universidad San Martín fue clasificado un $12,7 \%$, en la Universidad Libre fue clasificado el $81,3 \%$ y en la Universidad Central del Este fue clasificado el $88,3 \%$. En general, el modelo pudo clasificar el $61,2 \%$ correctamente.

\section{DISCUSIÓN}

En general, los resultados de los valores de orientación empática en los estudiantes de Medicina de las universidades estudiadas fueron diferentes, aunque las diferencias son pequeñas. No así los estudiantes de la misma carrera examinados en México ${ }^{13}$ y en Chile. ${ }^{22}$ Los puntajes observados en las mujeres resultaron diferentes de los de los hombres, coincidiendo con los de otras investigaciones realizadas en estudiantes y residentes de Medicina ${ }^{13,23}$ y estudiantes de Odontología, ${ }^{24}$ pero son contradictorios con otros estudios donde no se encontraron diferencias entre los sexos en distintas carreras relacionadas con las ciencias médicas. ${ }^{7,15,25-27}$ Sin embargo, existen antecedentes que señalan que las mujeres son más empáticas que los hombres; ;2,28 por lo tanto, habría que tratar de encontrar, en futuros estudios, las razones que expliquen estos resultados contradictorios, toda vez que la teoría muestra que hombres y mujeres son diferentes, incluso, en la estructura del cerebro, ${ }^{29}$

TABla 3. Resultados de la comparación múltiple de medias de Duncan de las sumatorias de los valores observados en cada universidad analizada

\begin{tabular}{llccc}
\hline & N & \multicolumn{3}{c}{ Subconjunto } \\
\cline { 3 - 4 } Universidad & & $\mathbf{1}$ & $\mathbf{2}$ & $\mathbf{3}$ \\
\hline Universidad Central del Este & 515 & 101,6893 & 103,8763 \\
Universidad San Martín & 283 & & & 107,5449 \\
Universidad del Norte & 345 & & & 107,6086 \\
Universidad Libre & 695 & 1,000 & 1,000 & 0,948 \\
Significancia & & & & \\
\hline
\end{tabular}

TABLA 4. Resultados de la comparación múltiple de medias de Duncan de las sumatorias de los valores observados en cada curso analizado

\begin{tabular}{lcccc}
\hline & & & \multicolumn{3}{c}{ Subconjunto } \\
\cline { 3 - 4 } Cursos & N & $\mathbf{1}$ & $\mathbf{2}$ \\
\hline Quinto año & 178 & 102,7640 & 103,7718 \\
Tercer año & 241 & 103,7718 & 104,3750 & 105,8492 \\
Cuarto año & 320 & 104,3750 & 105,8492 & 107,0092 \\
Segundo año & 557 & & & 0,311 \\
Primer año & 542 & 0,186 & 0,086 & \\
Significancia & & & & \\
\hline
\end{tabular}


y se conoce que las mujeres son más receptivas a las señales emocionales $\mathrm{y}$, por consiguiente, tienden más a medidas de apoyo emocional y entendimiento, lo cual contribuye a reforzar las relaciones empáticas. Los hombres se inclinan a ofrecer soluciones racionales..$^{23,30,31}$ AlcortaGarza y cols. ${ }^{13}$ proponen que se necesitan más pruebas que permitan relacionar las diferencias de empatía por género con factores intrínsecos (como la teoría evolutiva de inversión paternal) o factores extrínsecos (como la expectativa del rol de género), lo cual implicaría una actitud diferente para la selección y el entrenamiento en la carrera de Medicina.

No existe una distribución uniforme de la orientación empática entre los cursos de todas las universidades estudiadas. Esto resultados coinciden con los observados en otros estudios, en los que se ha demostrado una declinación de la orientación empática en estudiantes de
Odontología y Medicina a medida que estos transcurren por cursos cada vez más avanzados ${ }^{25}$ mientras que son contradictorios con los encontrados en el Reino Unido por Tavakol, Denning y Tavakol. ${ }^{32}$ Los hallazgos contradictorios entre las diferencias en el género, las universidades y los cursos apuntan a la presencia de otros factores, no necesariamente psicológicos, que podrían incidir en los niveles de orientación empática. En tal sentido, es posible plantear que dichos factores desconocidos podrían estar asociados a fenómenos sociológicos que influyen sobre la empatía. Esto se pone en evidencia en las diferencias observadas entre las universidades a partir del análisis discriminante.

En relación con la última idea presentada, se puede volver a citar las ventajas vinculadas a la presencia de empatía en el personal de salud para una mejor atención del paciente, ya reseñadas en la introducción..$^{9-12}$ De ahí que el desarrollo de

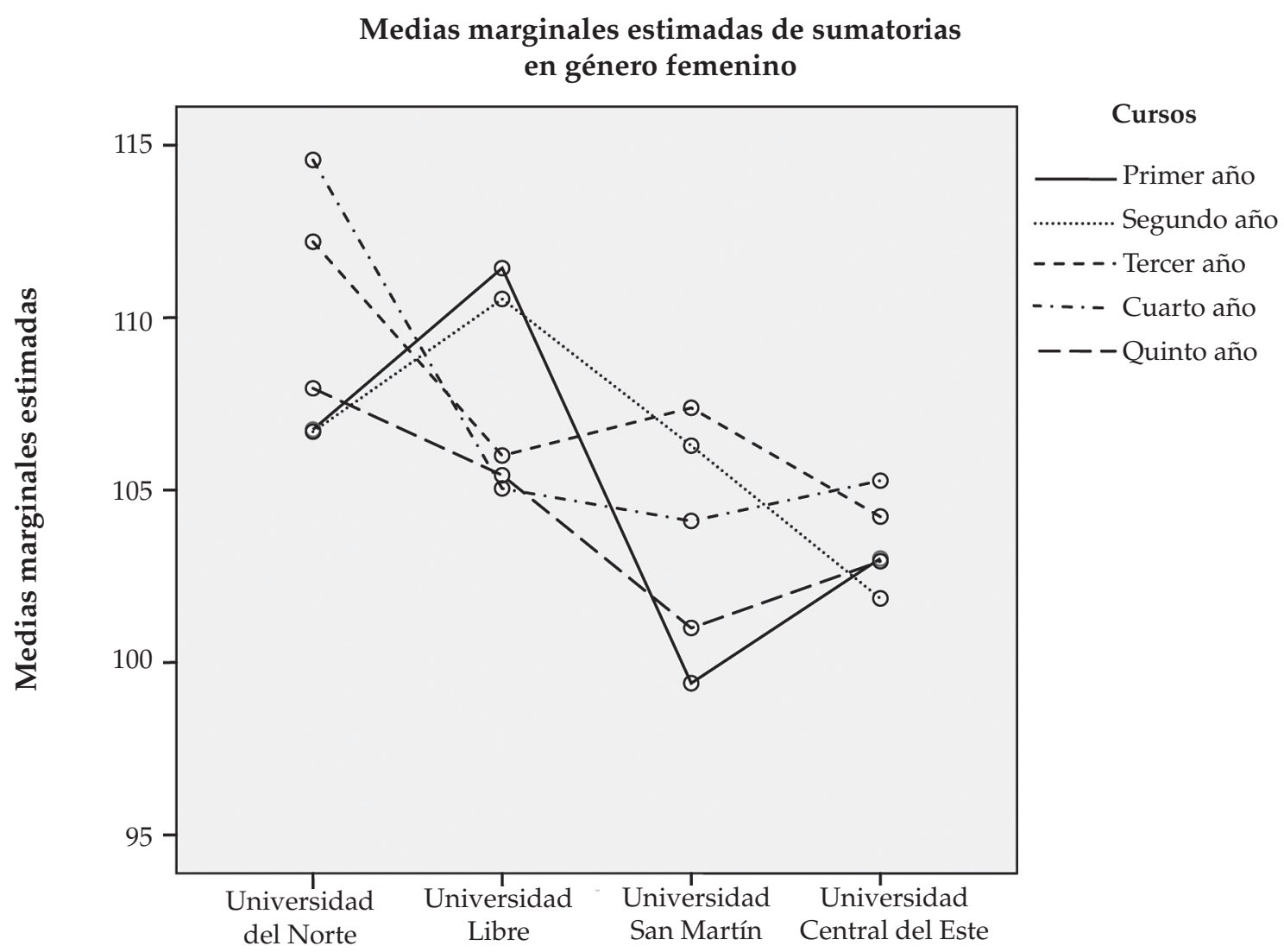

Universidad 
Figura 2. Perfil del comportamiento de las medias estimadas en las universidades y los cursos en los varones

Medias marginales estimadas de sumatorias en género: maculino

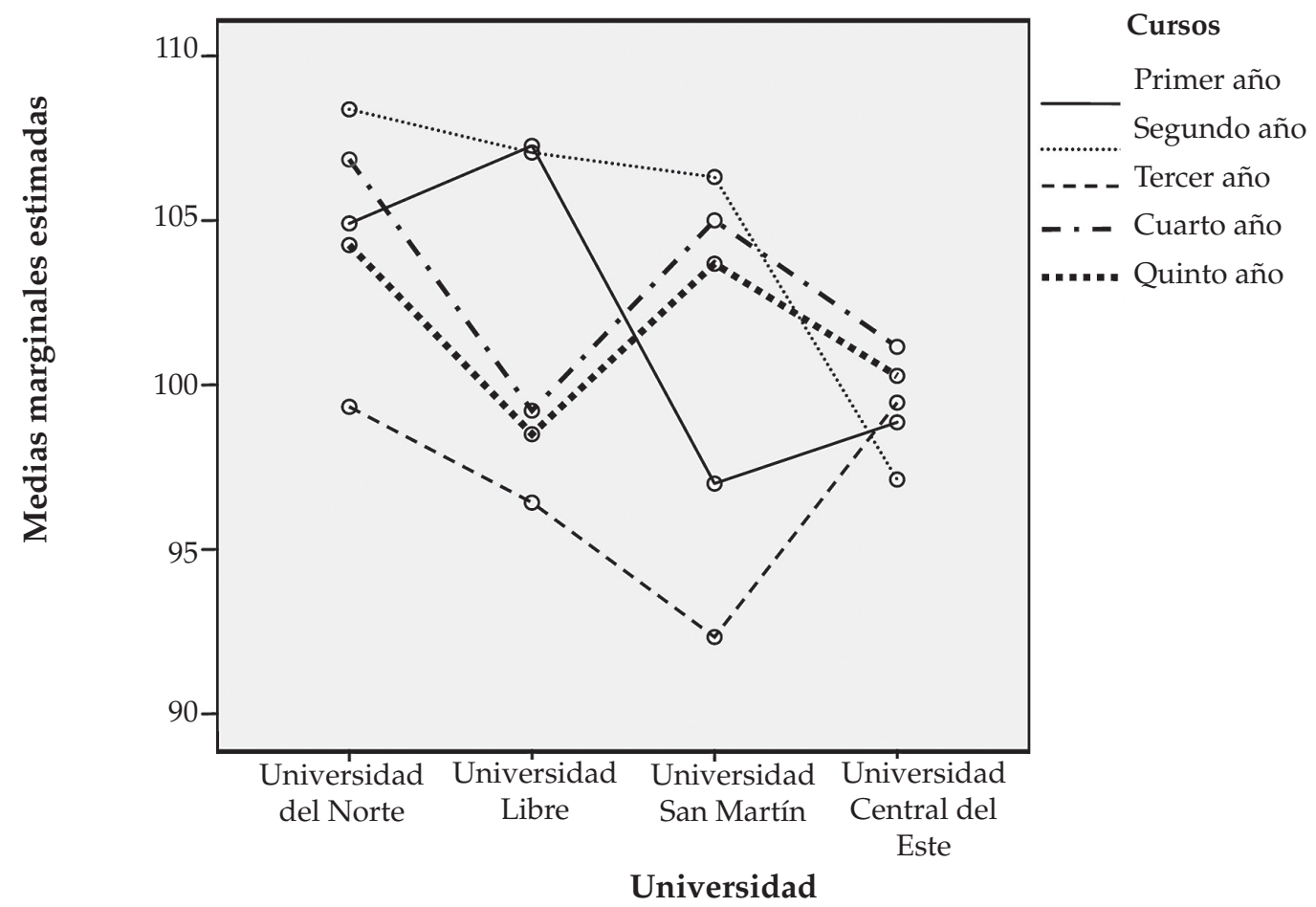

FIGURA 3. Centroides de grupo (universidades) y dispersión de los datos en torno a su respectivo centroide

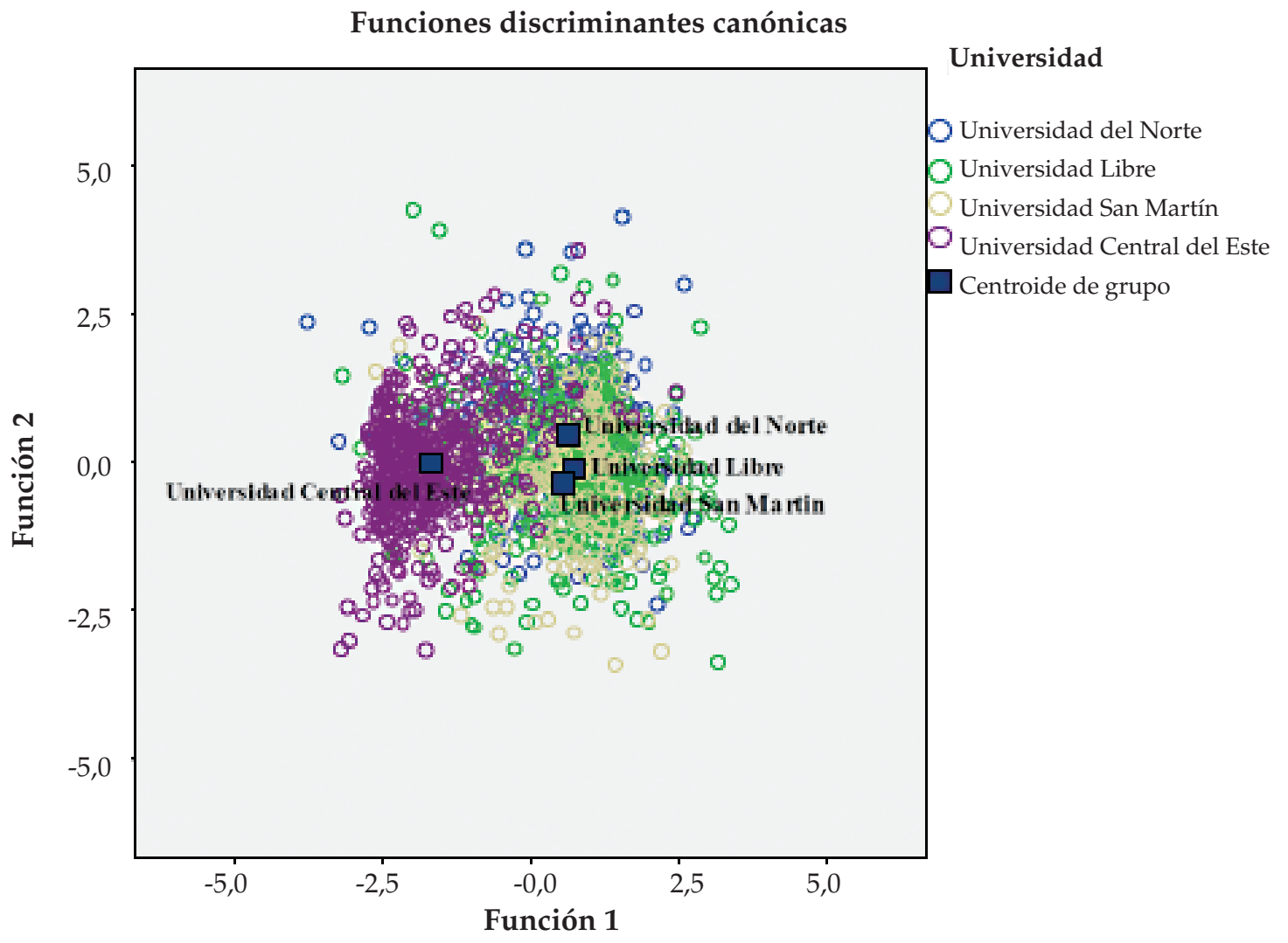


la empatía en los estudiantes de Medicina en el proceso de enseñanza-aprendizaje incidirá en el futuro en la forma de atención del paciente y en la calidad de la atención. Sin embargo, existen trabajos que ponen en duda la posibilidad de que la empatía pueda cambiar si se produce una intervención educacional, ${ }^{2,13}$ mientras otros autores reconocen que la empatía se puede aprender y que la relación médico-paciente, la posibilidad médica de ayudar a aliviar el dolor, el poseer conocimiento médico para ayudar, el médico en posición jerárquica en relación con el paciente son aspectos que parecen ser objeto de aprendizaje. ${ }^{13,33}$

Los factores, hasta hoy conocidos, que influyen en la empatía son:

a) la toma de perspectiva;

b) la fantasía,

c) la preocupación empática;

d) el malestar personal. ${ }^{2,33}$

Por otra parte, los enlaces entre cognición, lenguaje, experiencia familiar y empatía implican una conexión entre la sensibilidad a señales afectivas y el desarrollo de la cognición social, ${ }^{33}$ todo lo cual muestra que la empatía va muy unida a la cognición social y esta es dada desde el microsistema y el macrosistema donde se mueve el individuo, estableciéndose entre ambos una relación dialéctica. Esta última idea apoya la presencia de factores sociales a los cuales se ha referido en términos muy generales anteriormente. Por ejemplo, "en la etapa escolar y adolescencia la cultura del colegio influye en las capacidades empáticas de los estudiantes dando más oportunidades para que se ejercite la responsividad empática en la vida diaria". ${ }^{33} \mathrm{Al}$ respecto, se plantea que "se admite el supuesto de que la sensibilidad empática favorecerá probablemente el altruismo y reprimirá la agresión, pero que la persona realice o no acciones altruistas depende de otros determinantes, como los inductores sociales, las limitaciones que impone la situación, los costos potenciales, la disponibilidad de habilidades y de recursos necesarios para ayudar a otro, la atribución de responsabilidad, las características de la víctima y su relación con el observador". ${ }^{34}$ Por lo tanto, lo expresado apunta a que uno de los factores que influyen directamente en la aprehensión de la empatía es la "cognición social", ${ }^{35}$ la cual "incluye las áreas de procesamiento de emociones, la percepción social, el conocimiento de las reglas sociales, el estilo atribucional y la teoría de la mente".$^{35}$ Inducimos, entonces, que la cognición social es indispensable para el funcionamiento en comunidad. Como consecuencia, la empatía contribuiría directamente a este funcionamiento.

La empatía integra cognición, emoción y autorregulación. ${ }^{34}$ Como consecuencia, podría afirmarse que constituye una forma básica de comunicación social que puede ocurrir en contextos sociales diferentes y, por lo tanto, requiere la identificación de las respuestas emocionales en otras personas, e implica habilidades o competencias bien definidas. ${ }^{6}$

Las diferencias y similitudes observadas podrían explicarse por el "impacto" que pueden tener los componentes sociales sobre la empatía en los estudiantes de Medicina de las facultades de los países estudiados y constituiría un posible hallazgo empírico de la influencia que tendrían estos factores sobre la empatía. Esta podría

TABLA 5. Resultados de la clasificación de los sujetos en cada universidad de acuerdo con el modelo de análisis estadístico aplicado

\begin{tabular}{|c|c|c|c|c|c|c|c|}
\hline & & & & Grupo de & enencia pronos & & \\
\hline & & Universidad & $\begin{array}{c}\text { Universidad } \\
\text { del Norte }\end{array}$ & $\begin{array}{c}\text { Universidad } \\
\text { Libre } \\
\end{array}$ & $\begin{array}{l}\text { Universidad } \\
\text { San Martín }\end{array}$ & $\begin{array}{c}\text { Universidad } \\
\text { Central del Este }\end{array}$ & Total \\
\hline & & Universidad del Norte & 69 & 230 & 10 & 36 & 345 \\
\hline & $\overrightarrow{\tilde{c}}$ & Universidad Libre & 27 & 565 & 20 & 83 & 695 \\
\hline$\stackrel{\widetilde{G}}{0}$ & 己ु & Universidad San Martín & 18 & 187 & 36 & 42 & 283 \\
\hline 赵 & & Universidad Central del Este & 19 & 39 & 3 & 454 & 515 \\
\hline & & Universidad del Norte & 20,0 & 66,7 & 2,9 & 10,4 & 100,0 \\
\hline & $\%$ & Universidad Libre & 3,9 & 81,3 & 2,9 & 11,9 & 100,0 \\
\hline & & Universidad San Martín & 6,4 & 66,1 & 12,7 & 14,8 & 100,0 \\
\hline & & Universidad Central del Este & 3,7 & 7,6 & 0,6 & 88,2 & 100,0 \\
\hline
\end{tabular}

Clasificados correctamente el $61,2 \%$ de los casos agrupados originales. 
ser la explicación de las "cercanías" entre las facultades que pertenecen a una misma ciudad y a un mismo país en relación con la "lejanía" que manifiesta una facultad de otro país respecto de las anteriores, independientemente de si los valores de las sumatorias observadas al aplicar la escala EEJJ sean "bajos", en general, en todas las facultades evaluadas. Sin embargo, la pregunta: ¿cuáles son los componentes sociales "específicos" y "generales", y cómo actúan?, no puede ser respondida y explicada en el presente trabajo. A su vez, el hecho de que la empatía tenga un componente social permite justificar la tendencia teórica que plantea que puede estar relacionada con habilidades y competencias y, si es así, no solo la universidad y, concretamente, las facultades de Medicina, son las únicas responsables de ir incorporando estos aspectos en su currículo, sino que este es un problema que debe ser asumido también desde el ambiente familiar hasta el sistema educacional completo de un país. Sin embargo, lo antes planteado no elude la asunción de que la relación médico-paciente debe ser tomada en cuenta e incluida seriamente como una variable vital en los currículos en las facultades de Medicina, como parte de la responsabilidad social de las universidades de formar profesionales integrales, aprovechando la oportunidad "biológica" de que los estudiantes, en su mayoría, son jóvenes y su cerebro aún brinda ventanas de oportunidades evolutivas. Los resultados del presente trabajo apoyan el punto de vista de que la empatía es un "...concepto elusivo, difícil de definir y más aún de medir. Su multidimensionalidad y las propiedades internas de sus componentes la hacen menos aprehensible para los métodos de estudio e investigación tradicionales". Por lo tanto, es necesario seguir investigando cómo las relaciones específicas entre los factores componentes de la empatía se materializan de una forma concreta, en un lugar concreto, y el peso específico que tiene cada uno de ellos en la formación adecuada de un estudiante de Medicina.

\section{Agradecimientos}

A los Dres. Alberto Enrique D' Ottavio (Facultad de Ciencias Médicas, Universidad Nacional de Rosario, Argentina), Jaime Contreras (Facultad de Medicina, Universidad Andrés Bello, Chile); Peter Mc Coll (Facultad de Medicina, Universidad Andrés Bello, Chile), Hernán Borja Rebolledo (Director de la Carrera de Medicina, Universidad Andrés Bello, Viña del Mar); Mariano
Grilli (Facultad de Ciencias Médicas, Universidad de La Plata, Subsede Mar del Plata, Argentina); Felix Díaz Rodríguez (Facultad de Educación, Universidad Federal de Bahía, Brasil), Claudio Flores Würth (Facultad de Medicina, Universidad Austral de Chile), Verónica Hering (Facultad de Medicina, Universidad Austral de Chile), Sara Herrera Leyton (Facultad de Ciencias de la Salud, Universidad Católica del Maule, Chile), Gizaku Kuramochi (Universidad de la Frontera, Chile), Doris Carrasco Mardones (Universidad de Concepción, Chile), por sus acertadas sugerencias, las cuales fueron introducidas debidamente en el texto de este trabajo.

\section{BIBLIOGRAFÍA}

1. Hojat M, Gonnella JS, Nasca TJ, MangioneS, et al. Physician Empathy: Definition, Components, Measurement and Relationship to Gender and Specialty. Am J Psychiatry 2002;159(9):1563-9.

2. Fernández-Pinto I, López-Pérez B, Márquez M. Empatía: teorías y aplicaciones en revisión. An Psicol 2008;24 (2):284-98.

3. Sharmay-Tsoory SG, Tormer R, Goldsher D, Berger BD, et al. Impairment in Cognitive and Affective Empathy in Patiens with Brain Lesions: Anatomical and Cognitive Correlates. J Clin Exp Neuropsychol 2004;26 (8):1113-27.

4. Elliot R, Völlm B, Drury A, McKie S, et al. Co-operation with another player in a financially rewarded guessing game activates regions implicated in theory of mind. Social Neurosc 2006;1(3-4):385-95.

5. Davis MH, Hull JG, Young RD, Warren GG. Emotional Reactions to Dramatic Film Stimuli: The Influence of Cognitive and Emotional Empathy. J Pers Soc Psichol 1987;52(1):126-33.

6. Fernández-BerrocalP,Extremera N. Emotional intelligence: A theoretical and empirical review of its first 15 year of history. Psicothema 2006;18:7-12.

7. Hojat M, Mangione S, Nasca TJ, Cohen MJM, et al. The Jefferson Scale of Empathy: development and preliminary psychometric data. Educ Psychol Measur 2001;61(2):346-65.

8. Gallangher HL, Frith CD. Functional imaging of "theory of mind". Trends Cogn Sci 2003 Feb;7(2):77-83.

9. BeckmanHB, FranckelRM. Theeffect of physician behaviour on the collection data. Ann Inter Med 1984;101(5):692-6.

10. Falvo D, Tippy P. Communicating information to patients: patient satisfaction and adherence as associated with resident skill. J Fam Pract 1998;26(6):643-7.

11. Barsky AJ 3rd. Hidden reasons why some patients visit doctors. Ann Int Med 1981;94(4-1):492-8.

12. Levinson W, Roter DL, MulloolyJP,DullVT, etal. Physicianpatient communication: the relationship with malpractice claims among primary care physicians and surgeons. JAMA 1997;277(7):553-9.

13. Alcorta-Garza A, González-Guerrero JF, Tavitas-Herrera SE, Rodríguez-Lara FJ, et al. Validación de la Escala de Empatía Médica de Jefferson en Estudiantes de Medicina Mexicanos. Salud Ment 2005;28(5):57-63.

14. Mangione S, Kane GC, Caruso JW, Gonnella JS, et al. Assessment of empathy in different years of internal medicine training. Med Teach 2002;24(4):371-4.

15. Rojas AM, Castañeda Barthelemiez S, Parraguez-Infiesta RA.Orientación empática de los estudiantes de dos escuelas 
de kinesiología de Chile. Educ Méd 2009;12(2):103-9.

16. Shapiro SS, Wilk MB. An Analysis of Variance Test for Normality (Complete Samples). Biometrika 1965;52(34):591-611.

17. Hair JF, Anderson RE, Tatham RL, Black WC. Análisis multivariante. Madrid: Prentice-Hall; 2001. Págs.66-7.

18. Díaz-Narváez VP. Metodología de la Investigación Científica y Bioestadística para Profesionales y Estudiantes de Ciencias Médicas. Santiago, Chile: RiLEditores; 2009. Págs.415-41.

19. Frías MD, Llobell JP, García JF. Tamaño del efecto del tratamiento y significación estadística. Psicothema 2000;2(2):236-40.

20. Levy JP, Varela J. Análisis Multivariante para las Ciencias Sociales. Madrid: Pearson Prentice Hall; 2003.Págs.249-57.

21. Visauta B. Análisis estadístico con SPSS. Vol II. Madrid: McGraw-Hill; 1998.Pags.135-7.

22. Carvajal A, Miranda CI, Martinac T, García C, et al. Análisis delnivel de empatía en un curso de quinto año de medicina, a través de una escala validada para este efecto. Rev Hosp Clín Univ Chile 2004;15(4):302-6.

23. Hojat M, Gonella JS, Mangione S, Nasca TJ, et al. Empathy in medical students as related to academic performance, clinical competence and gender. Med Educ 2002;36(6):522-7.

24. Sherman J, Cramer A. Measurement of Changes in Empathy During Dental School. J Dent Educ 2005;69(3):338-45.

25. Hojat M, MangioneS, Nasca TJ, RattnerS, etal. An empirical study of decline in empathy in medical school. Med Educ 2004;38:934-41.

26. Rivera I, Arratia R, Zamorano A, Díaz-Narváez VP. Evaluación del nivel de orientación empática en estudiantes de Odontología. Salud Uninorte, Barranquilla 2011;27(1):63-72.

27. Hojat M, Gonnella JS, Nasca TJ, Mangione S, et al. The Jefferson scale of physician empathy: further psychometric data and difference by gender and speciality at item level. Acad Med 2002;77(10):58-60.

28. Retuerto PA. Diferencias en empatía en función de las variables género y edad. Apuntes de Psicología 2004;22(3):323-39.

29. Baron-Cohen S. La gran diferencia: Cómo son realmente los cerebros de hombres mujeres. Barcelona: Amat; 2005. Págs.15-24.

30. Hojat M, Gonella JS, XU G. Gender comparisons of young physicians' perceptions of their medical education, professional life, and practice: a follow up study of Jefferson Medical College graduates. Acad Med 1995;70:305-12.

31. Ickes W. Gender differences in empathic accuracy: Differential ability or differential motivation? Methodology Meta Analysis, Social Perception and Cognition 2006;7(1):95109.

32. Tavacol S, Dennick R, Tavacol M. Empathy in UK medical y year and specialty interest. Education for Primary Care 2011;22:297-303.

33. Almonte C, Montt MA. Psicopatología infantil y de la adolescencia. Santiago, Chile: $2^{\mathrm{a}}$ Ed. Mediterráneo; 2012. Págs.45-58.

34. Mestre MV, Samper P, Frías MD. Procesos cognitivos y emocionales predictores de la conducta prosocial y agresiva: la empatía como factor modulador. Psicothema 2002;4(2):227-32.

35. Moya-Albiol L, Herrero N, Bernal MC. The neural bases of empathy. Rev Neurol 2010;50(2):89-100. 


\section{Anexo 1}

Estimado Estudiante:

La evaluación del paciente respecto de la calidad de la atención clínica está determinada por los conocimientos científicos y tecnológicos del profesional y por otros factores, entre ellos, la empatía.

Por lo anterior surge la necesidad de estudiar la empatía en los estudiantes de Medicina. Para ello estamos realizando la investigación denominada: "Medición del nivel de orientación empática en alumnos de Facultades o Carreras de Medicina".

Esta investigación NO perjudica a ningún estudiante.

Esta investigación SÍ tiene grandes beneficios para todos los estudiantes porque les permitirá contribuir significativa y directamente a la posibilidad de mejorar su curriculum o pensum. Por tanto, su contribución es decisiva.

Para este fin se les adjunta una encuesta que posee las siguientes características:

1. No debe escribir su nombre, esta es una encuesta confidencial.

2. Responda en forma individual.

3. Todas las respuestas son posibles. No existe preconcepción para responderlas.

4. No existen respuestas correctas o incorrectas, sino diferentes estilos de respuestas, por tanto cualquier respuesta es válida y aporta a la investigación.

5. Está en una escala de 1 a 7. En la encuesta se explica la valoración.

6. Si Ud. no entiende algún enunciado, pregunte al examinador.

7. Es necesario responder a todas las preguntas.

\section{Le damos las gracias por su contribución al desarrollo de la investigación.}

\section{ESCALA DE EMPATÍA MÉDICA DE JEFFERSON (VERSIÓN - S)}

Curso: $1^{\circ} \_2^{\circ} \_3^{\circ} \_4^{\circ} \_5^{\circ} \_$Género: $\mathrm{M} \_$F_ Edad

Indique si ha reprobado alguna asignatura: Sí__

Instrucciones: Indique su grado de acuerdo o desacuerdo con cada una de las siguientes afirmaciones encerrando en un círculo el número apropiado después de cada afirmación. Utilice la escala de 7 puntos (un mayor número indica un mayor acuerdo):

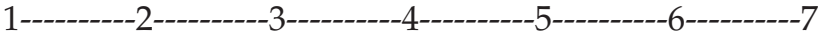

Totalmente en desacuerdo Totalmente de acuerdo

1. La comprensión que tenga el médico de los sentimientos del paciente y de su familia no influye en el tratamiento.

2. Los pacientes se sienten mejor cuando el médico comprende sus sentimientos.

$\begin{array}{lllllll}1 & 2 & 3 & 4 & 5 & 6 & 7 \\ 1 & 2 & 3 & 4 & 5 & 6 & 7 \\ 1 & 2 & 3 & 4 & 5 & 6 & 7 \\ 1 & 2 & 3 & 4 & 5 & 6 & 7 \\ 1 & 2 & 3 & 4 & 5 & 6 & 7\end{array}$

3. Es difícil para el médico ver las cosas desde la perspectiva de su paciente.

4. La comprensión del lenguaje corporal es tan importante como la comunicación verbal en la relación médico-paciente.

5. El buen sentido del humor del médico contribuye a tener un mejor resultado clínico. 
6. Debido a que la gente es diferente, es difícil ver las cosas desde el punto de vista del paciente.

7. Prestar atención a las emociones del paciente no es importante durante la anamnesis o historia clínica.

8. Prestar atención a las experiencias personales del paciente no influye en los resultados del tratamiento.

9. Los médicos deberían tratar de ponerse en el lugar del paciente cuando lo atienden.

10. Los pacientes valoran que el médico entienda sus sentimientos, lo cual es terapéutico por sí mismo.

11. La enfermedad del paciente puede ser curada solamente por el tratamiento médico; por lo tanto, los lazos emocionales del médico con su paciente no tienen influencia significativa en el tratamiento médico.

12. Preguntarle a los pacientes acerca de sus vivencias personales, no es de ayuda para comprender sus dolencias físicas.

13. Los médicos deberían tratar de comprender lo que está sucediendo en la mente de los pacientes, poniendo atención a la comunicación no verbal $\mathrm{y}$ al lenguaje corporal.

14. Yo creo que las emociones no tienen lugar en el tratamiento de la enfermedad médica.

15. La empatía es una habilidad terapéutica sin la cual el éxito del médico puede estar limitado.

16. La comprensión que tenga el médico del estado emocional de su paciente, así como también de su familia, es un componente importante de la relación médico-paciente.

17. Los médicos deberían pensar como sus pacientes para poder darles un mejor cuidado.

18. Los médicos no deberían dejarse influenciar por lazos personales fuertes entre sus pacientes y los miembros de sus familias.

19. No disfruto leer literatura no médica o de arte.

20. Creo que la empatía es un importante factor terapéutico en el tratamiento médico.

\begin{tabular}{|c|c|c|c|c|c|}
\hline 1 & 2 & 3 & 4 & 5 & 6 \\
\hline 1 & 2 & 3 & 4 & 5 & 6 \\
\hline 1 & 2 & 3 & 4 & 5 & 6 \\
\hline 1 & 2 & 3 & 4 & 5 & 6 \\
\hline 1 & 2 & 3 & 4 & 5 & 6 \\
\hline 1 & 2 & 3 & 4 & 5 & 6 \\
\hline 1 & 2 & 3 & 4 & 5 & 6 \\
\hline 1 & 2 & 3 & 4 & 5 & 6 \\
\hline 1 & 2 & 3 & 4 & 5 & 6 \\
\hline 1 & 2 & 3 & 4 & 5 & 6 \\
\hline 1 & 2 & 3 & 4 & 5 & 6 \\
\hline 1 & 2 & 3 & 4 & 5 & 6 \\
\hline 1 & 2 & 3 & 4 & 5 & 6 \\
\hline 1 & 2 & 3 & 4 & 5 & 6 \\
\hline 1 & 2 & 3 & 4 & 5 & 6 \\
\hline
\end{tabular}

